\title{
The Heart as a Bi-directional Scalar Field Antenna
}

\section{Oschman $\mathrm{JL}^{*}$ and Oschman NH}

Nature's Own Research Association, Dover, New Hampshire, USA

A discovery must be, by definition, at variance with existing knowledge.

Albert Szent-Györgyi [1]

Great spirits have always encountered opposition from mediocre minds. The mediocre mind is incapable of understanding the man who refuses to bow blindly to conventional prejudices and chooses instead to express his opinions courageously and honestly.

$\sim$ Albert Einstein [2]

The above quotes pertain to some of the investigators and concepts discussed in this report. New vistas in biology and medicine lie before us if we are willing to recognize our biases, blind spots, and unsolved problems. Science and medicine cannot progress when new ideas are rejected without thorough and appropriate investigation. Simply denying the existence of unfamiliar phenomena is a much easier path to follow, and is often the method of choice of the professional skeptics. As an example of taking the easy path, Oliver Heaviside and others replaced Maxwell's original quaternions with vector algebra, and eliminated the potential fields as "arbitrary" and unnecessary. Faraday's and Maxwell's potentials were thought to be purely mathematical entities without physical significance, but this is not so in quantum physics, as was demonstrated by Aharonov and Bohm in 1959. By the end of the 1800's the Maxwell equations had been reduced from the original 20 to the 4 we find in physics texts today. Deleting the potentials deprived physics, biology and medicine of important theoretical tools for nearly a century. The long-neglected potentials have important implications for biology and medicine.

To summarize this article ${ }^{1}$, meticulous research conducted by an innovative Spanish cardiologist, Francisco Torrent-Guasp, revealed that the ventricle of the heart is a double helical structure, now known as the helical ventricular myocardial band (HVMB). Further work by Guasp and others revealed that this three-dimensional ventricular architecture can be regarded as a geometrically non-orientable surface, similar to a triple-twisted Möbius strip. This is one of several geometric arrangements used to document the Aharonov-Bohm effects, involving electromagnetic vortical potential or scalar fields. Extraordinary yet compelling evidence has also indicated that the heart's energy fields, generated by vortical electrical flows through the heart and aortic arch, are coupled to fields of information that are not bound by the limits of time and space. These findings provide a potential basis for welldocumented intuitive or clairvoyant phenomena. They also support the controversial "morphic resonance" concept of Rupert Sheldrake, who has suggested that memory is inherent throughout nature and that organisms inherit, via a field phenomenon, a collective memory from all previous things of their kind. These concepts also relate to so-called "paranormal" topics such as precognition, as well as unconventional explanations of previously unsolved mysteries in biology such as the sources of the information involved in development, evolutionary inheritance, memory, and the quantum pupillary response.

In the early 1980's the first author was looking for research on electrical circuits within living things and found a fascinatingsounding reference to an article entitled The Electrical Circulation by Francisco Torrent-Guasp [3]. The reference was in the $35^{\text {th }}$ edition of

${ }^{1}$ The article is dedicated to the memory of Francisco Torrent-Guasp (1931-2005).
Gray's Anatomy, edited by Peter Williams and Roger Warwick [4]. A request for the article was made to the interlibrary loan librarian at the Marine Biological Laboratory (MBL) in Woods Hole, Massachusetts, USA. After some delay the librarian reported that the article could not be found anywhere in the USA. She suggested writing to the editors of Gray's Anatomy to ask if they had any further information about the article. Professor Peter Warwick from Guy's Hospital Medical School, University of London responded that Dr. Guasp came to London from time to time, and that he would be happy to communicate about his work. He provided Dr. Guasp's address, which was in Denia, Alicante, Spain. A note to Dr. Guasp led to an enthusiastic response consisting of a collection of reprints, some in English and some in Spanish. On the reprint of his Electrical Circulation article Dr. Guasp had written an effusive note expressing profound appreciation for attention to his work. His main discovery was that a blunt dissection of the heart revealed that the ventricle is a double helical structure, now known as the helical ventricular myocardial band (HVMB). A video on the Internet shows Dr. Guasp performing the dissection and unrolling the myocardial band [5].

Dr. Guasp was obviously enthusiastic that an American scientist had expressed interest in his work. At the time, he was not getting much attention for his concepts among cardiologists, but this has changed significantly. Pub Med now lists 50 articles since 1966 from a search for "Torrent-Guasp." Dr. Guasp is co-author on 30 of them and the remaining 20 are by other researchers who have continued his investigations. Two articles have already been published in 2015. Dr. Guasp also sent a silicone-rubber model of the helical heart. This model was developed by Torrent-Guasp, Whimster and Redmann in 1997 [6]: "The ventricular myocardial mass consists of a band, curled in a helical way, which extends from the pulmonary artery to the aorta. This is illustrated by a silicone rubber model cast from an actual unrolled myocardial band."

Guasp's discoveries and his dissection are deceptively simple - he actually invested half century of his life in painstaking and meticulous research before he was able to dissect the heart in a manner that unraveled the 'Gordian Knot' that had been a profound mystery of heart anatomy for almost five centuries [7].

Paco Torrent-Guasp's life ended suddenly in 2005 in Madrid after he gave the closing lecture at a meeting on electrophysiology and arrhythmias organized by Jerónimo Farré and Concha Moro. Professor Guasp had been very pleased with the atmosphere at the conference, the way he had been treated, the attention and respect he had been

*Corresponding author: James L Oschman, Nature's Own Research Association, New Hampshire, USA, Tel: 603-742-3789; E-mail: joschman@aol.com

Received August 26, 2015; Accepted September 24, 2015; Published October 02, 2015

Citation: Oschman JL, Oschman NH (2015) The Heart as a Bi-directional Scalar Field Antenna. Fluid Mech Open Acc 2: 121. doi:10.4172/2090-8369.1000121

Copyright: (C) 2015 Oschman JL, et al. This is an open-access article distributed under the terms of the Creative Commons Attribution License, which permits unrestricted use, distribution, and reproduction in any medium, provided the original author and source are credited. 
shown both by organizers and participants at the conference. He was delighted that he had been able to explain some his pioneering theories about the electric circulation [8]. Details of the anatomy of the HVMB are summarized in Figure 1 [9].

We have used Guasp's silicone-rubber model of the helical heart many times in lectures and workshops to demonstrate the double helical structure of the HVMB, and to introduce the possibility of resonance between vortical heart fields and double helical DNA molecules in cells throughout the body. Evidence for heart/DNA resonance was reported in 1993 by Rein and McCraty [10]. Rein had already demonstrated that quantum fields (electromagnetic potentials, A-fields, or scalar fields) can influence neurological [11] and immunological functions [12]. Cyril Smith et al. in the UK documented effects of magnetic A-fields in both allergic responses and in the effects of homeopathic remedies [13], concluding that chemicals and their electromagnetic signatures produce identical effects.

Glen Rein thought DNA would make a good target for testing healers' ability to affect biological systems, since well-established quantitative measures of DNA's conformational state existed and offered a stable and reliable measuring system. He had tested this model system with several healers by having them hold test tubes containing DNA while they attempted to create a healing environment, and had obtained some positive indicators that the conformational state of DNA changed under these conditions.

In 1991, Dr. Rein continued these studies at the HeartMath Research Center in Boulder Creek, California. A number of experiments with DNA were conducted over a period of a year and a half. The first six months were primarily spent performing a series of control studies to insure the stability of the measurement system and refining the protocols. Doc Childre from the Institute then added the element of

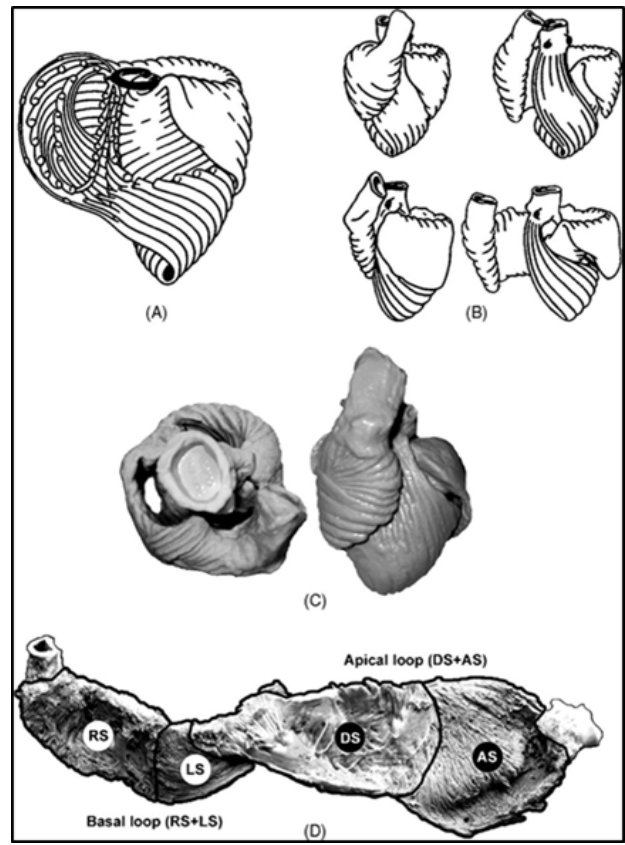

Figure 1: $(A)$ is a copy of the last drawing made by Torrent-Guasp, illustrating the complex three-dimensional fiber architecture of the ventricular mass. The schematic of the silicone-rubber mould of the HVMB is shown in (B), and his silicone-rubber mould of the HVMB is shown at (C); The segmental anatomy of the HVMB is shown in (D): RS, right segment; LS, left segment; DS descendent segment; AS, ascendant segment. The illustration is Figure 9 from Kocica et al. [9]. intentionality to the protocols, which proved to be a key factor.

Rein's resonance hypothesis was confirmed in two studies at the Institute of HeartMath in collaboration with Rollin McCraty [14,15]. These studies demonstrated (a) coherence in the electrocardiographic (ECG) frequency spectra of individuals whose attention was focused in the heart area while generating positive emotions such as love, care or appreciation and (b) a correlation between the ECG coherent patterns and those also occurring in the brain and other parts of the body. F-A Popp's demonstration of quantum coherence in biological systems [16] supported the concept of a coherent endogenous electro-magnetic field within the body. Taken together, these results strengthened the HeartMath Institute's theory that the heart acts as a master electrical oscillator capable of radiating coherent frequencies which promote the health and vitality of the entire human system [17]. The theory also proposed that physiological benefits of coherent heart frequencies produced during positive emotional states are mediated through DNA. In 1996 Russek and Schwartz [18] suggested that the heart can be viewed as a dynamical energy-generating system, and coined the term "energy cardiology".

Further research by McCraty et al. led to extraordinary and compelling evidence that the heart's energy field is coupled to a field of information that is not bound by the limits of time and space. Rigorous experimental studies demonstrated that the heart and brain receive and process information about an event before the event actually happens $[19,20]$. Moreover, the physiological data showed that the heart appears to receive "intuitive" information a few seconds before the brain. The researchers suggested that the heart is directly coupled to an ambient subtle energetic field of information that surrounds the body, which, in turn, is entangled and interacts with the multiplicity of energy fields in which the body is embedded, presumably including fields present in the quantum vacuum.

Taken together, these findings support the controversial "morphic resonance" concept of Rupert Sheldrake, who has suggested that memory is inherent in nature and that organisms inherit a collective memory from all previous things of their kind [21]. To summarize Sheldrake's thesis: morphogenetic fields give rise to all living forms; matter assumes form when it resonates with a field; morphogenetic fields are derived from an organism's own past actions and from the structures and actions of ancestors; and these fields act across space and time. If verified, these are profoundly important concepts. They resolve one of the profound outstanding mysteries in biology: the origin of form in organisms.

The recent investigations of the HVMB model of the heart show that the three-dimensional ventricular architecture can be regarded as a geometrically and mathematically non-orientable surface, similar to triple-twisted Möbius strip (Figure 2). This is one of several geometric configurations of self-cancelling coils used to document AharonovBohm effects [22,23] involving electromagnetic potential or scalar fields. The Möbius strip or Möbius band is a surface with only one side and only one boundary. The Möbius strip was discovered independently by the German mathematicians August Ferdinand Möbius and Johann Benedict Listing in 1858. A Möbius strip can be constructed from a three-dimensional rectangle. Twisting this rectangle (i.e. a paper-strip) for $180^{\circ}$ (one or more times) and attaching the ends to one another seems to eliminate one dimension of three dimensional space (Figure 2). This possibility correlates with the curved space - time continuum or Minkowski space, discussed by Einstein in his special theory of relativity.

Extraordinary as these conclusions may be, they are supported by 


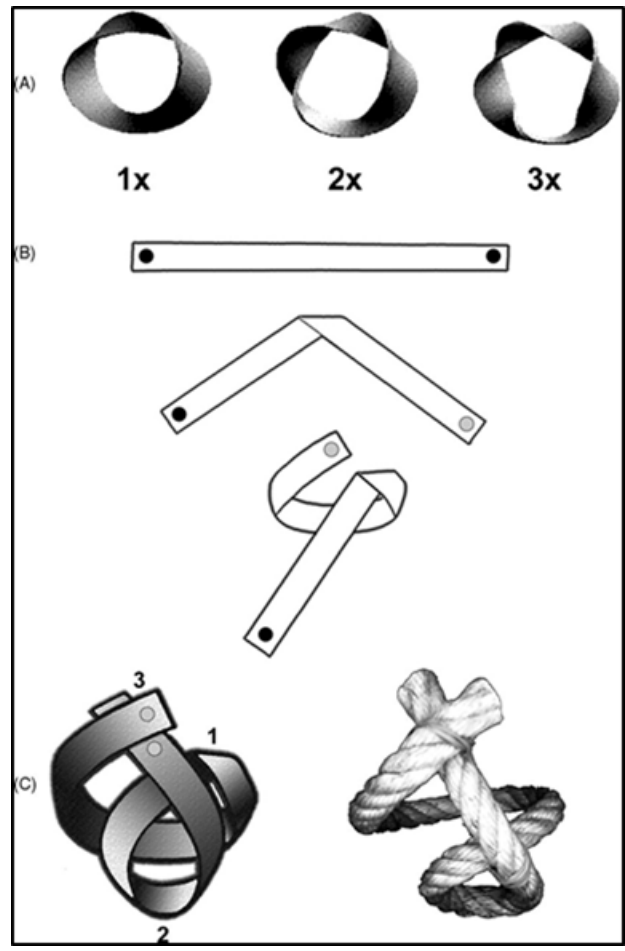

Figure 2: The central fold of the HVMB is demonstrated with paper-strip (A). In (B) a black dot is on the visible side and a gray dot is on the invisible side of the strip. (C) shows paper-strip and helical rope models of the HVMB with annotated sites of three principal spirals within a complex helicoid: 1, centra fold; 2, apical loop; 3 , great arteries (i.e. 'truncus arteriosus' spiral septation). The illustration is Figure 10 from Kocica et al. [9]. Reproduced by permission of Dr. Manel Ballester-Rodés.

other evidence of biological effects of potential fields. For example, in 1994, Ho et al. reported that such fields can alter pattern formation in Drosophila embryos [24]. They viewed embryos as possible detectors of the Aharonov-Bohm effect, thereby providing a possible basis for the influence of morphogenic fields as discussed above.

An experiment by scientists at the Australian National University, using helium atoms scattered by light, has blurred the distinction between past, present and future [25]. In these studies, cause and effect were reversed. The future events actually caused past events. Professor Andrew Truscott summarized the studies: "A future event causes the photon to decide its past".

These findings and conclusions are also consistent with the syntropy concept developed by one of the major mathematicians of the 20th century, the Italian Luigi Fantappiè (1901-1956). His concept was based on the recognition that phenomena produced by past events (causes) are governed by the 2nd law of thermodynamics, entropy (energy dissipates, order decreases, structures disintegrate), whereas phenomena that will occur in the future (goals, also called attractors) are governed by a symmetrical law, which he referred to as syntropy [26]. In 1940, Fantappiè noticed that the equation of D'Alembert [27], which governs the propagation of waves, had two solutions: a solution describing waves diverging from a source, and a solution describing waves converging towards a receiver. Fantappiè's derivations were based on the equations of relativistic and quantum physics, including the discovery that in quantum physics all laws are symmetrical with respect to time. In 1974, Albert Szent-Györgyi referred to syntropy as "negative entropy." This is a process by which forms tend to evolve toward higher levels of organization, order, and dynamic harmony [28]. He also referred to this as, "the drive in nature to perfect itself." Anticipating problems with the term "drive", he stated:

I know that many of my colleagues, especially the molecular biologists, will be horrified, if not disgusted, to hear me talk about a "drive" and will call me a "vitalist," which is worse than to be called a communist. But I think that the use of such words as "drive" does no harm if we do not imagine we have found an explanation by finding a name. If we look upon such words as simply denoting great unsolved problems of science, they can even lead to useful experimentation.

Finally, Davis [29] has demonstrated a new pupillary response in the eye which appears to occur prior to cognitive processing. The pupillary response occurs before mental processing begins, without stimulus recognition and without visual perception of the stimulus. As in the study of McCraty et al. $[19,20]$ the pupils constricted to positive images and responded with dilation to negative images, whether or not the images were visually available.

One of the most significant implications of these discoveries is their potential application to consciousness research. Conventional thinking is that the brain is the seat of consciousness and memory. Evidence summarized here opens the possibility that as a scalar field antenna the heart might be able to tap into so called "empty" space, which appears to contain structure and energy and information in coherent holographic form [30-32].

Many modern thinkers are drifting away from purely mechanistic explanations of how the universe and those within it function, as well as the nature of consciousness ${ }^{2}$. The results on the structure of the heart, described here, along with the scalar and vortex concepts of Meyl [33-35] point to softer and more holistic and even spiritual quantum perspectives of an interconnected universe in which potential waves connect all things. As a bi-directional Möbius structure ${ }^{3}$, the human heart seems suited for linking holographic organisms to each other and to a holographic environment, and vice versa, via scalar fields. For a fascinating discussion between physicist David Bohm, who developed the concept of the implicate order, and biologist Rupert Sheldrake who proposed the morphogenetic field concepts [36].

Examples include neurosurgeon Karl H. Pribram, quantum theoretical physicist David Bohm, biologist Rupert Sheldrake, anesthesiologist Stuart Hameroff, engineer and astronaut Edgar Mitchell, endocrinologist Depak Chopra, cosmologist and philosopher Richard L. Amoroso, philosopher Ervin László, the inventor of holography Dennis Gabor, mathematical physicist Roger Penrose, astrophysicist Rudolph E. Schild, and developer of BodyTalk John Veltheim.

${ }^{3}$ From basic antenna theory we know that an antenna of a particular geometry will function for both transmission and reception of electromagnetic waves and, presumably, of potential waves.

\section{References}

1. Szent-Gyorgyi A (1972) Dionysians and Apollonians. Science 176: 966.

2. Einstein A (1940) Quoted from the New York Times.

3. Guasp FT (1970) The electrical circulation. Guasp, Denia

4. Warwick PL, Williams R (1973) Gray's Anatomy (35thedn), British 1399

5. Watch Spanish cardiologist Dr. Francisco Torrent Guasp dissect a cow heart to reveal the helical structure of the ventricular myocardial band.

6. Torrent-Guasp FF, Whimster WF, Redmann K (1997) A silicone rubber mould of the heart. Technol Health Care 5: 13-20.

7. Pettigrew JB (1864) On the arrangement of the muscular fibres in the ventricles of the vertebrate heart, with physiological remarks. Phylos Trans 154: 445-500.

8. Aguilar JC (2005) In Memoriam. Francisco Torrent-Guasp (1931-2005) Rev Esp Cardiol 58: 759

9. Kocica MJ, Corno AF, Carreras-Costa F, Ballester-Rodes M, Moghbel MC, et 
Citation: Oschman JL, Oschman NH (2015) The Heart as a Bi-directional Scalar Field Antenna. Fluid Mech Open Acc 2: 121. doi:10.4172/20908369.1000121

al. (2006) The helical ventricular myocardial band: blobal, three-dimensional, functional arthitecture of the ventricular myocardium. Eur J Cardiothorac Surg 29: $21-40$

10. Rein G, McCraty R (1993) Modulation of DNA by coherent heart frequencies. In: Proceedings of the Third Annual Conference of the International Society for the Study of Subtle Energy and Energy Medicine, Monterey, California 58-62.

11. Rein G (1993) Modulation of neurotransmitter function by quantum fields In: Behavioral Neurodynamics. Pribram KH (eds.) Internat. Neural Network Society, Washinngton DC.

12. Rein $\mathrm{G}$ (1991) Utilization of a cell culture bioassay for measuring quantum fields generated from a modified caduceus coil. Proc. $26^{\text {th }}$ Intersoc Energy Convers Engineer Conf Boston, USA.

13. Smith CW (1987) Electromagnetic effects in humans. In: Fröhlich $\mathrm{H}$ ed Biological Coherence and Response to External Stimuli. Springer-Verlag, Berlin 14: 205-232.

14. Rein G, McCraty R (1993) Local and nonlocal effects of coherent heart frequencies on conformational changes of DNA. In: Proceedings of the Joint USPA/IAPR Psychotronics Conference, Milwaukee. Wisconsin.

15. Rein G, McCraty R (1994) Structural changes in water and DNA associated with new physiologically measurable states. Journal of Scientific Exploration 8: $438-439$.

16. Popp FA (1979) Photon storage in biological systems. In: Electromagnetic Bikoinformation. Popp FA et al eds. Urban \& Schwarzenberg, München.

17. Childre DL (1992) Self Empowerment: The heart approach to stress management. Planetary Publications, Boulder Creek, CA USA.

18. Russek LG, Schwartz GE (1996) Energy Cardiology: A Dynamical Energy Systems Approach for Integrating Conventional and Alternative Medicine, Advances: The Journal of MindBody Health 12: 4-24.

19. McCraty R, Atkinson M, Bradley RT (2004) Electrophysiological evidence of intuition: Part 1. The surprising role of the heart. Journal of Alternative and Complementary Medicine 10: 133-143.

20. McCraty R, Atkinson M, Bradley RT (2004) Electrophysiological evidence of intuition: Part 2. A system-wide process? Journal of Alternative and Complementary Medicine 10: 325-336.

21. Sheldrake R (1995) A New Science of Life: The Hypothesis of Morphic Resonance. Park Street Press, South Paris, ME Paris.
22. Aharonov $Y$, Bohm D (1959) Significance of electromagnetic potentials in quantum theory. Physical Review 115: 485-491.

23. Batelaan H, Tonomura A (2009) The Aharonov-Bohm effects: Variations on a subtle theme. American Institute of Physics, College Park, MD. Physics Today 62: $38-43$.

24. Ho MW, French A, Haffegee J, Saunders PT (1994) Can weak magnetic fields (or potentials) affect pattern formation? Chapter 7 in Ho MW, Popp FA Warnke U (eds.), Bioelectrodynamics and biocommunication. World Scientific Singapore.

25. Manning AG, Khakimov RI, Dall RG, Truscott AG (2015) Wheeler's delayedchoice gedanken experiment with a single atom. Nature Physics 11: 539-542.

26. Fantappie $L$ (1942) Sull interpretazione. The Unified Theory of the Physical and Biological World, Published in Spanish in 1943. 4: 7.

27. D'Alembert JLE (1747) Articles extraits de I'Histoire de l'Académie des Sciences et Belles-Lettres de Berlin, Berlin.

28. Szent-Györgyi A (1974) Drive in living matter to perfect itself. Synthesis 1: 14-26.

29. Davis CE (2007) The quantum field pulpillary response.

30. Ho MW, el Naschie M, Vitiello G (2015) Is Spacetime Fractal and Quantum Coherent in the Golden Mean? Global Journal of Science Frontier Research $1-20$.

31. Maldacena JM (1998) The Large N Limit of Superconformal Field Theories and Supergravity. Adv Theor Math Phys 2: 231-252.

32. Cowen R (2013) The Universe Really Is a Hologram, According to New Simulations. Nature magazine.

33. Meyl K (2003) Scalar Waves: From an extended vortex and field theory to a technical, biological and historical use of longitudinal waves. INDEL GmbH, Verlagsabteilung, Fillingen-Schwennigen, Germany.

34. Meyl K (2012) About vortex physics and vortex losses. Journal of Vortex Science and Technology 1: 1-10.

35. Meyl K (2012) DNA and Cell Resonance: Magnetic Waves Enable Cel Communication. DNA and Cell Biology 31: 422-426.

36. Bohm D, Sheldrake R (1982) Morphic Fields and the Implicate Order, A dialogue with David Bohm. 